\title{
Research Paper \\ The Effect of Mental Imagery on Preoperative Anxiety of Elective Hernia
}

\author{
Narjes Heshmatifar ${ }^{1} \odot$, Mahboobeh Mohebbi ${ }^{2} \odot{ }^{*}$ Fatemeh Borzoee $^{3} \odot$, MohammadHasan Rakhshani $^{4} \odot$
}

1. Department of Nursing, Sabzevar University of Medical Sciences, Sabzevar, Iran.

2. Department of Nursing, Imam Reza Hospital, Mashhad University of Medical Sciences, Mashhad, Iran.

3. Department of Operating Room, Sabzevar University of Medical Sciences, Sabzevar, Iran.

4. Department of Biostatistics, Faculty of Medicine, Sabzevar University of Medical Sciences, Sabzevar, Iran.

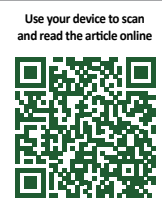

Cftation: Heshmatifar N, Mohebbi M, Borzoee F, Rakhshani M. [The Effect of Mental Imagery on Preoperative Anxiety of Elective Hernia (Persian)]. Complementary Medicine Journal. 2020; 9(4):3930-3939. https://doi.org/10.32598/cmja.9.4.954.1

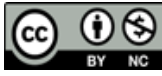

Article Info:

Received: 31 Oct 2019

Accepted: 24 Dec 2019

Available Online: 01 Mar 2020

Keywords:

Mental imagery

Anxiety, Surgery,

Complementary

medicine

\section{A B STRACT}

Objective Preoperative anxiety is associated with adverse effects such as fatigue, nausea, vomiting, agitation, increased demand for analgesics, and prolonged postoperative hospitalization. This study aimed to investigate the impact of mental imagery on anxiety in patients undergoing hernia repair surgery. Methods In this randomized clinical study, after obtaining informed written consent, 56 patients for hernia surgery were randomized into two experimental and control groups. In the experimental group, guided imagery was applied. Data was collected using the State-Trait Anxiety Inventory (STAI). The collected data was analyzed using SPSS V. 20.

Results The average age of participants was $30.8 \pm 11.22$ years, and $73 \%$ of them were male. The average of STAI level in guided imagery group before intervention were $41.42 \pm 6.75$ and $32.22 \pm 9.87$, respectively, which decreased to $33.07 \pm 4.31$ and $28.14 \pm 7 / 86$ after intervention. On the other side, the average of STAI level in the control group were $39.71 \pm 4.58$ and $29.68 \pm 8.49$, respectively, which increased to $44.60 \pm 6.44$ and $38.42 \pm 7.21$ at the end of the study. Statistically, the paired t-test showed a significant difference between the levels of anxiety before and after study in two groups $(P<0.001)$.

Conclusion This study cleared that performing preoperative mental imagery can reduce the STAI level in patients before surgery.

\section{Extended Abstract}

\section{Introduction}

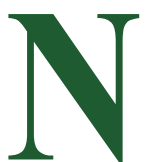

o matter how minor the surgery is, it remains an anxiety factor for the patient, so that each patient may consider their surgical operation day as the most threatening day of their lives [2]. Anxiety reduction as a caring approach should be considered for each patient [7]. The treatments currently used to reduce anxiety mainly focus on pharmacological interventions, while some medi- cations cause adverse reactions and delay post-operative waking and discharge [8]. In recent years, the emphasis has been on non-pharmacological methods to reduce anxiety [9]. Non-pharmacological measures due to their noninvasive and inexpensive nature can reduce anxiety, stop or reduce drug use, and increase safety. Mental imagery as part of complementary medicine is a mind-body technique [10]. This technique does not require specialized equipment or extensive training, and the patient is easily trained and readily accepted [11]. Since there are conflicting results from the impact of mental imagery on anxiety, it further highlights the importance of doing research in this regard.

* Corresponding Author:

Fatemeh Borzoee, MSc.

Address: Department of Operating Room, Sabzevar University of Medical Sciences, Sabzevar, Iran.

Tel: +98 (51) 44011000

E-mail: borzoee75026@yahoo.com 


\section{Materials and Methods}

The present study is a randomized clinical trial that was performed on 56 patients before their hernia surgery. After completing the informed consent form, patients were selected based on accessibility and divided into intervention and control groups using randomized permuted blocks (6part blocks). The sample size was 30 persons in each group. The demographic questionnaire and Spielberger's State and Trait Anxiety Inventory (STAI) were used for data collection. One hour before going to the operating room, questionnaires were completed after initial selection of research units. Finally 28 people remained in each group. In the intervention group, in addition to routine care, an audio file containing relaxing music and visualization of beautiful scenery (seashore), along with positive affirmations related to increased health and repetition of calming words such as "God", "love" and "beauty" aired through headphones for 18 minutes an hour before surgery.

The control group received only routine care and anxiety was again measured after 18 minutes. At the end of the study, the Spielberger questionnaire was completed in both groups. Data were analyzed by SPSS V. 20 and statistical tests used were Mann-Whitney, chi-square, paired t-test, independent $t$-test and Analysis of Variance (ANOVA).

\section{Results}

The mean age of the subjects was $30.8 \pm 17.28$ in the intervention group and $32.9 \pm 14.18$ in the control group. Findings of the study showed that there was no statistically significant difference between the two groups in terms of age, sex, marital status and educational level.

Mean pre and post-intervention anxiety in intervention group was $41.42 \pm 6.75$ and $32.22 \pm 9.87$ respectively, which decreased to $33.07 \pm 4.31$ and $28.14 \pm 7.86$ after intervention, respectively. In the control group, they were $39.71 \pm 4.58$ and $29.68 \pm 8.49$, which at the end of the study increased to $44.60 \pm 6.44$ and $38.42 \pm 7.21$, respectively. This difference was statistically significant $(\mathrm{P}<0.001)$ (Table 1$)$.

Before intervention, there was no significant difference between the two groups regarding the level of manifest anxiety $(\mathrm{P}=0.187)$. However, there was a significant difference after intervention $(\mathrm{P}=0.001)$ (Table 2).

\section{Discussion}

The findings of the present study showed that preoperative mental imagery reduces anxiety in the intervention group compared to the control group.

Results of the study by Gonzales et al showed that this method was effective in reducing preoperative anxiety [21];

Table 1. Comparison of mean score of anxiety in research units in mental imagery and control group

\begin{tabular}{ccccc}
\hline \multirow{2}{*}{ Group } & Anxiety & \multicolumn{2}{c}{ Mean \pm SD } & P \\
\cline { 3 - 5 } & & Before Intervention & After Intervention & Paired t-test \\
\hline \multirow{2}{*}{ Mental imagery group } & manifest & $41.42 \pm 6.75$ & $33.07 \pm 4.31$ & $P<0.001$ \\
& hidden & $32.22 \pm 9.87$ & $28.14 \pm 7.86$ & $\mathrm{P}<0.001$ \\
\hline Control group & manifest & $39.71 \pm 4.58$ & $44.60 \pm 6.44$ & $\mathrm{P}<0.001$ \\
& hidden & $29.68 \pm 8.49$ & $38.42 \pm 7.21$ & $\mathrm{P}<0.001$ \\
\hline
\end{tabular}

Table 2. Mean anxiety before and after the intervention in two groups of mental imagery and control

\begin{tabular}{cccc}
\hline Variable & Mental Imagery Group & Control Group & P \\
\hline Manifest anxiety & Mean \pm SD & Mean $\pm S D$ & independent t-test \\
Before intervention & $41.42 \pm 6.75$ & $39.71 \pm 4.58$ & $\mathrm{P}=0.187$ \\
After intervention & $33.07 \pm 4.31$ & $44.60 \pm 6.44$ & $\mathrm{P}<0.001$ \\
\hline
\end{tabular}


but in their study of the control group - unlike the present study - there was no significant difference between baseline and preoperative anxiety, which is an expected phenomenon with respect to approaching the time of surgery.

In a review study, Hadjibalassi et al. reported that mental imagery was effective in improving the physical and mental health outcomes of patients in the intensive care unit [22]. In the study of Beyza'i et al., mental imagery has been shown to reduce anxiety, depression, and stability of vital symptoms in patients undergoing hemodialysis, which is consistent with the results of the present study [23].

In contradiction with the results of the current study, Thomas et al. reported the ineffectiveness of mental imagery in reducing postoperative anxiety [24]. Discrepancies between the results of these two studies can be attributed to reasons such as the research method; also the Thomas study had only post-test. The number of women in the intervention group was higher than the control group, so that this difference was significant. Also, the selection of participants in the intervention group was non-random. The results of the study by Urech et al. also showed no significant difference between mental imagery compared to muscle relaxation on reducing anxiety in pregnant women [25]. This difference may be due to the sample size $(n=13)$ and the duration of the intervention (10 minutes). In general, these findings suggest a more parasympathetic activity that changes the physiological responses and subsequently, patients feel more relaxed and less anxious [21].

Mental imaging can be used as an easy, inexpensive and non-invasive way to reduce patients' anxiety before surgery.

\section{Ethical Considerations}

Compliance with ethical guidelines

This article was extracted from a research project approved by Sabzevar University of Medical Sciences with code of ethics MEDSAB.REC.92.1. It was also registered at the Iranian Clinical Trial Registration Center with the code IRCT2013122315905N1.

Funding

Sabzevar University of Medical Sciences financialy supported this study.

\section{Authors' contributions}

Article designing, sampling and compilation: Narjes Heshmatifar, Mahboubeh Mohebbi and Fatemeh Borzouei; Statistical issues: Mohammad Hassan Rakhshani.

\section{Conflicts of interest}

There is no conflict of interest in this study.

\section{Acknowledgements}

We are grateful to the research deputy of Sabzevar University of Medical Sciences, management of Shahid Beheshti Hospital, and its general surgery department staffs. 


\section{تأثير تصويرسازى ذهنى بر اضطراب قبل از عمل جراحى فتق انتخابى}

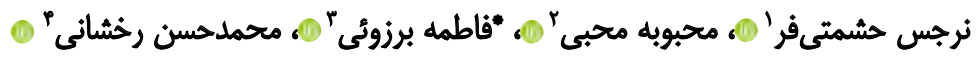

1. كروه يرستارى، دانشكده يرستارى، دانشكاه علوميزشكى سبزوار، سبزوار، ايران.

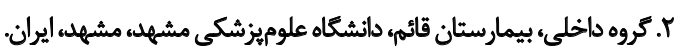

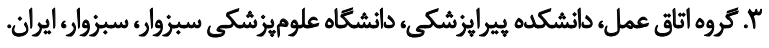

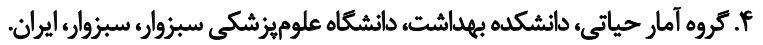

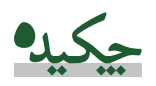

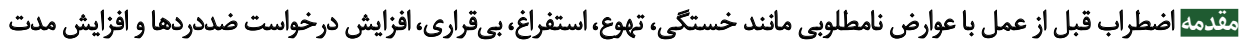

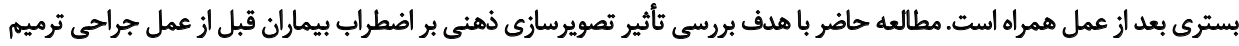
هرنى انجام شد.

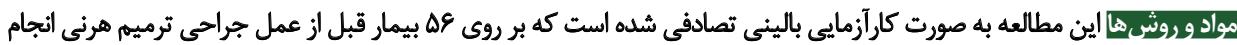

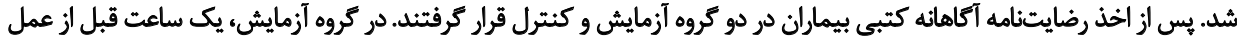

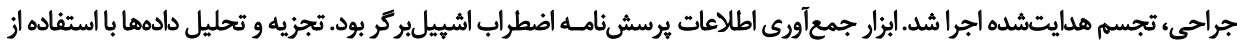

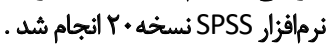

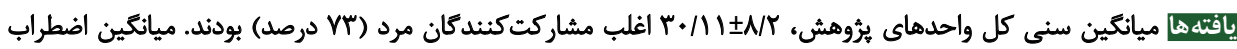

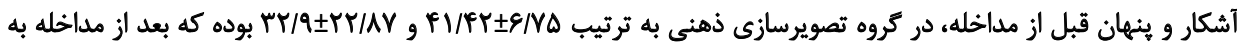

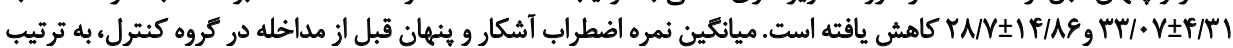

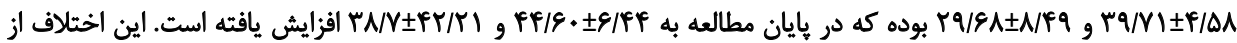

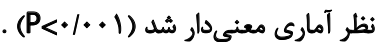

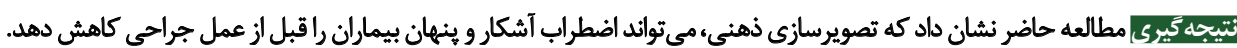

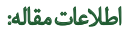

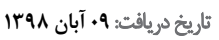
تاريخ يذيرش: ب. دى

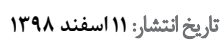

كليدوأرهها:

تصويرسازى ذهنى، اضطراب، جراحى، طب ذبن مكمل

بايد براى هر بيمار به عنوان يك رويكرد مراقبتى در نظر ترفته

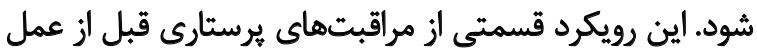

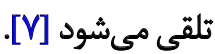

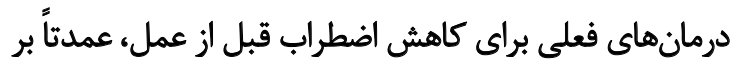

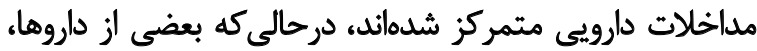

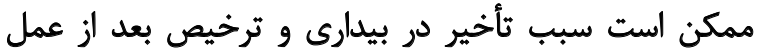
شوند. كاهى اوقات هم واكنش نامطلوب بات به داري داروها وجود داري دارد

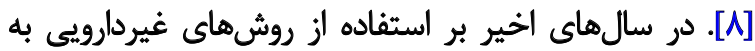

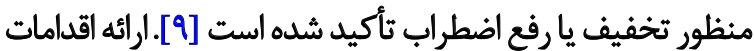

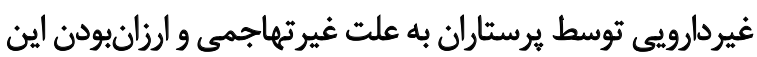

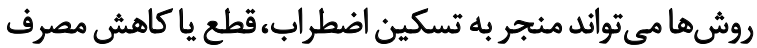

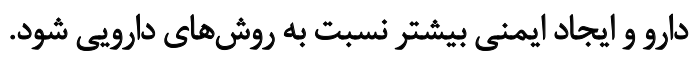

رابهجايى و بيرونزدكى احشا از يك ديواره باز ضعيفشده

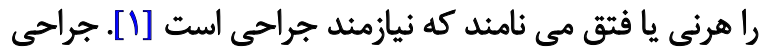

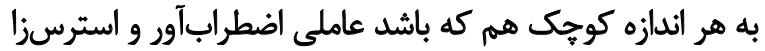

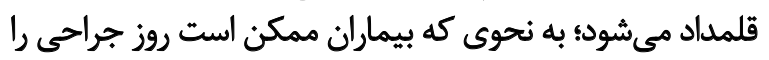

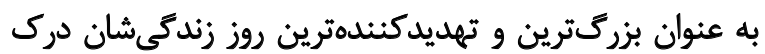

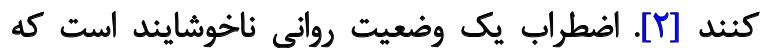

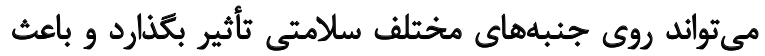

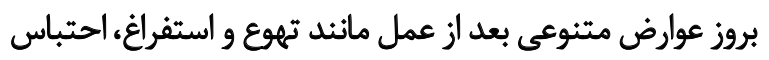

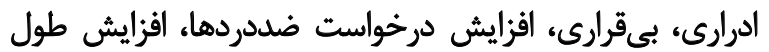

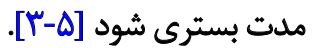

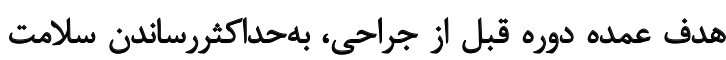

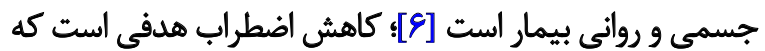

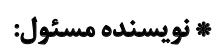

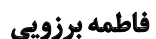
نشاني: سبزوار، دانشعاه علوميزشكى سبزوار، دانشكده بيرايزشكي، كروه اتأق عمل.

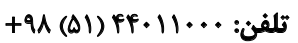
يست الكترونيكى: borzoee75026@yahoo.com 


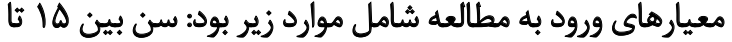

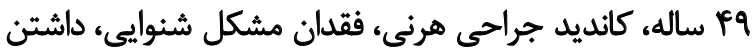

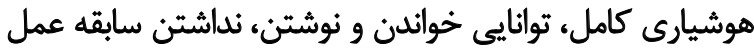

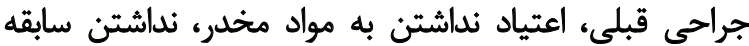

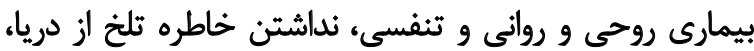

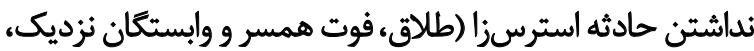

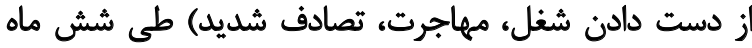

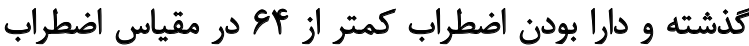

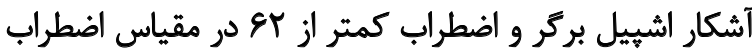

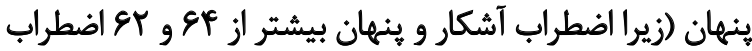

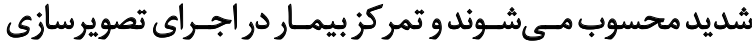

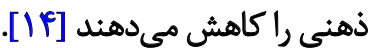
معيارهاى خروج شامل رضايت نداشتن بيمار به ادامه مشاركت

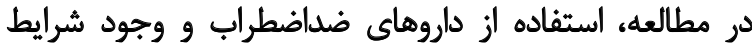
اورزانسى مانيد فتق مختنق بود. در اين تحقيق براى جمعآورى دادهها از دو برسشنامسه زير

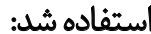

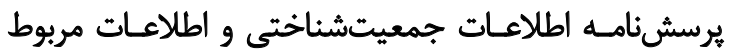

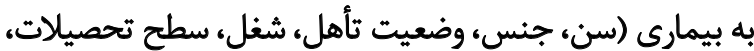

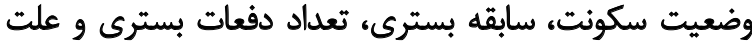

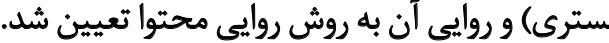

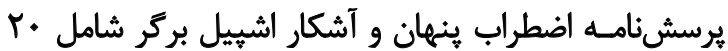

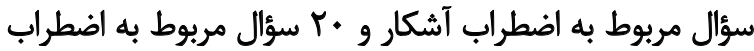

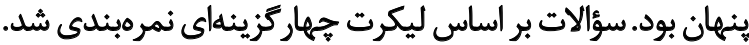

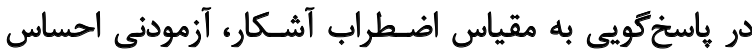

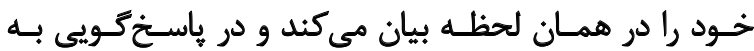

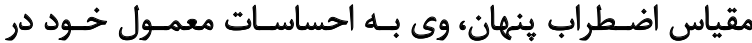

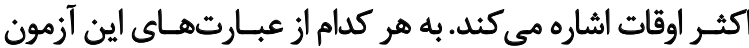

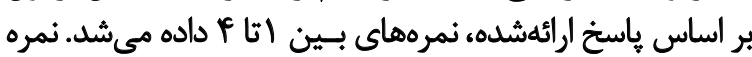

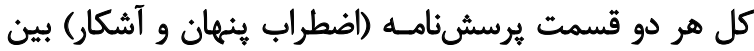

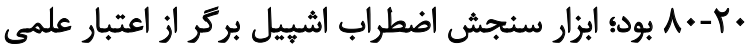

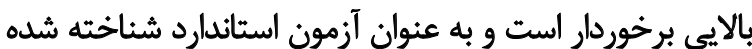

است [10]. - الايت برخودار

روايى آن را براى اولين بار، مهرام و همكاران در ايران به تأييد

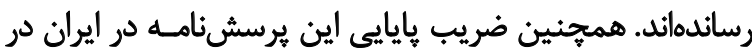

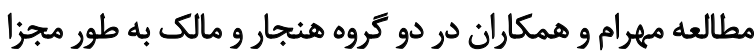

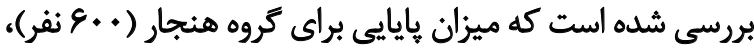

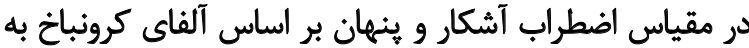

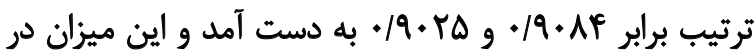

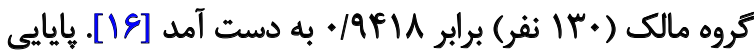

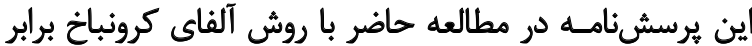

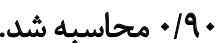

تصويرسازي ذهنى به عنوان بخشى از طب مكمل و جايكزين'،

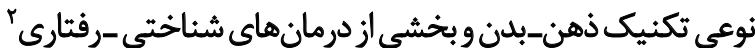

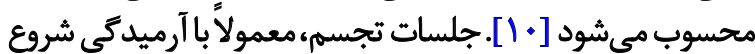

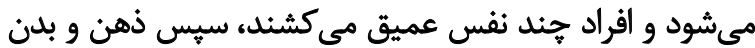

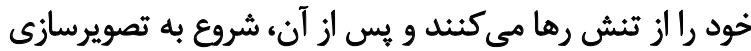

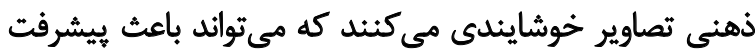

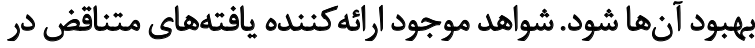

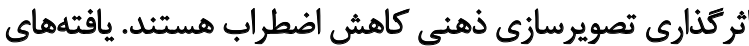

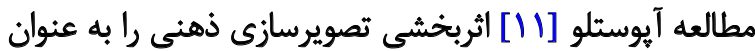

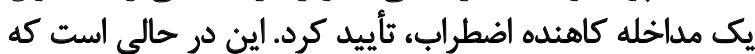

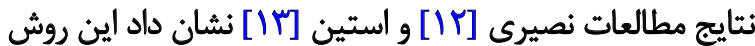
در كاهش اضطراب بيماران مؤثر نبوده است. شيوع فراوان اضطراب قبل از عمل و عواقب ناشى از آن،

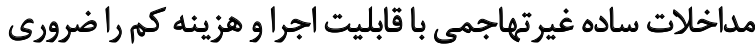

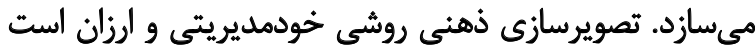

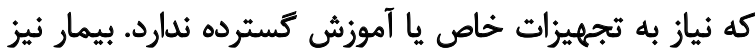

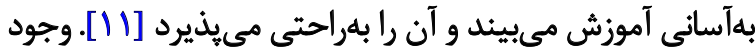

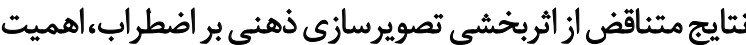

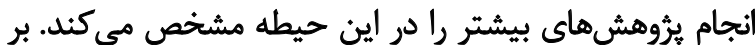

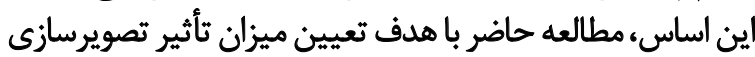

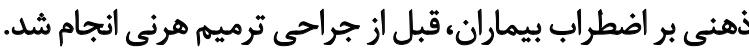

\section{مواد وروشها}

مطالعه حاضر از نوع كارآزمايى بالينى تصادفى شده است

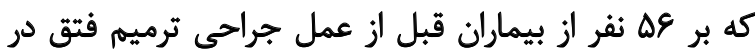

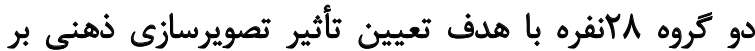

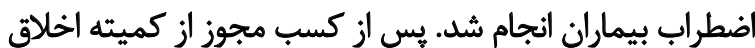

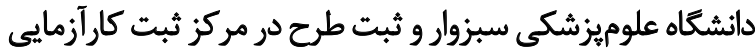

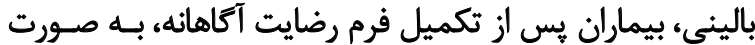

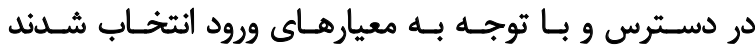

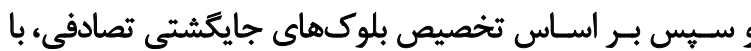

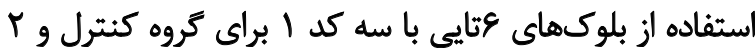

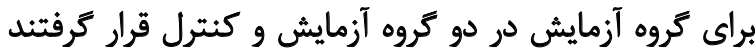

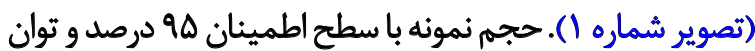

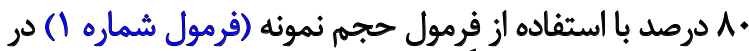

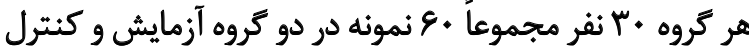
تعيين شد.

$$
n=\frac{2\left(Z_{1 \frac{a}{2}}+Z 1-\beta\right)^{2} s d^{2}}{\left(\mu \_1-\mu \_2\right)^{2}}=\frac{2(1.96+0.84)^{2}(0.4)^{2}}{(0.204)^{2}} 60
$$

1. Complementary or Alternative Medicine(CAM)

2. Cognitive-Behavioral Therapies (CBT) 


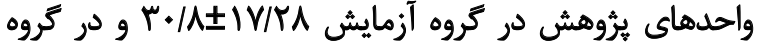

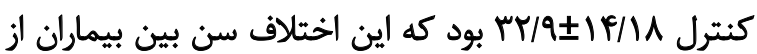

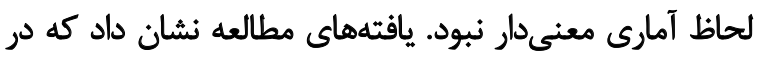

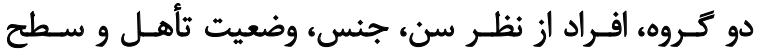

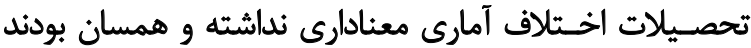

$$
\text { (جدول شماره 1). }
$$

تمام مشاركت كنئدكان (·• (1 درصد) حالتى از اضطراب رادر

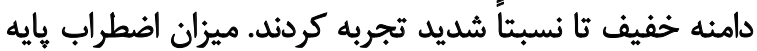

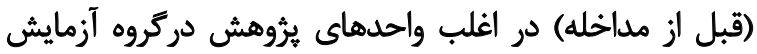

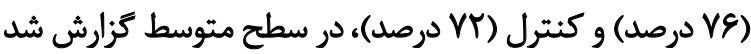

$$
\text { (تصوير شماره r). }
$$

همانطور كه در جدول شماره r نشان داده شده است

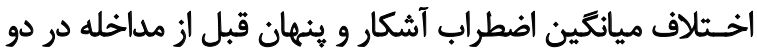

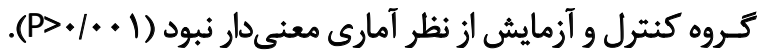
ميانكين اضطراب آشكار و ينهان قبل ازئ مداخله، در كروه تصويرسازى ذهنى به به ترتيب

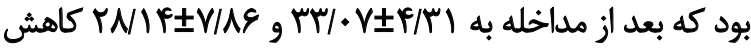
يافت. آزمون آمارى تى زوجى، بين ميزان اضطراب آناب آشكار و ينهان

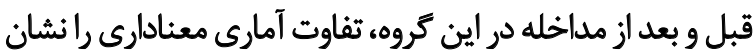

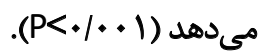

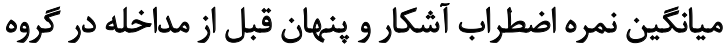
كنترل، به ترتيب ني اضطراب

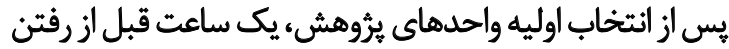

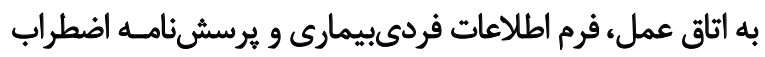

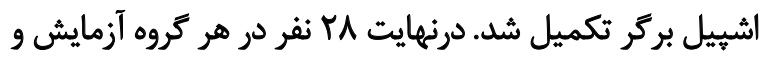

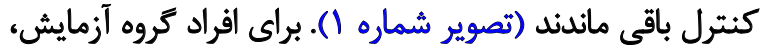

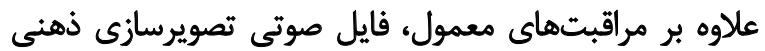

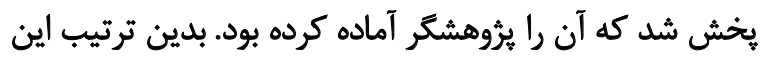

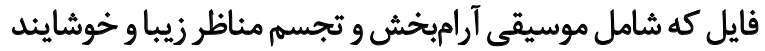

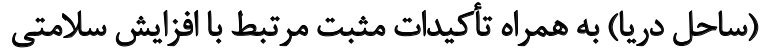

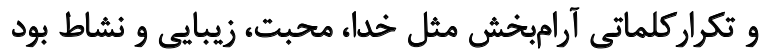

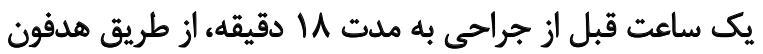

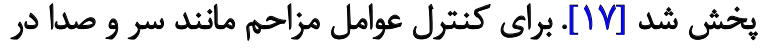

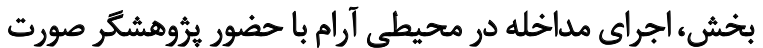

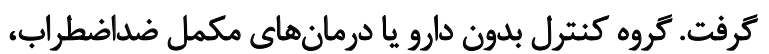

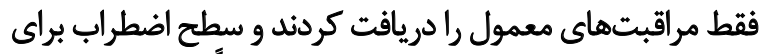

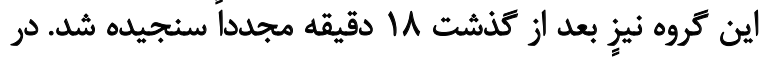

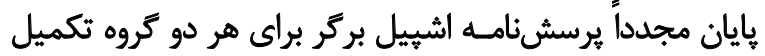

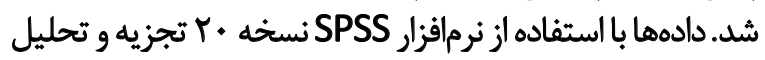

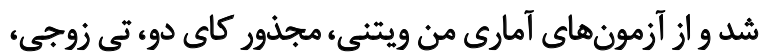
تى مستقل و آناليز واريانس استفاده شد.

يافتهاهl

تعداد كل واحدهاى يُؤهش عه نفر بور بود كه دو كروه كنترل

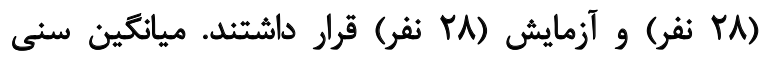

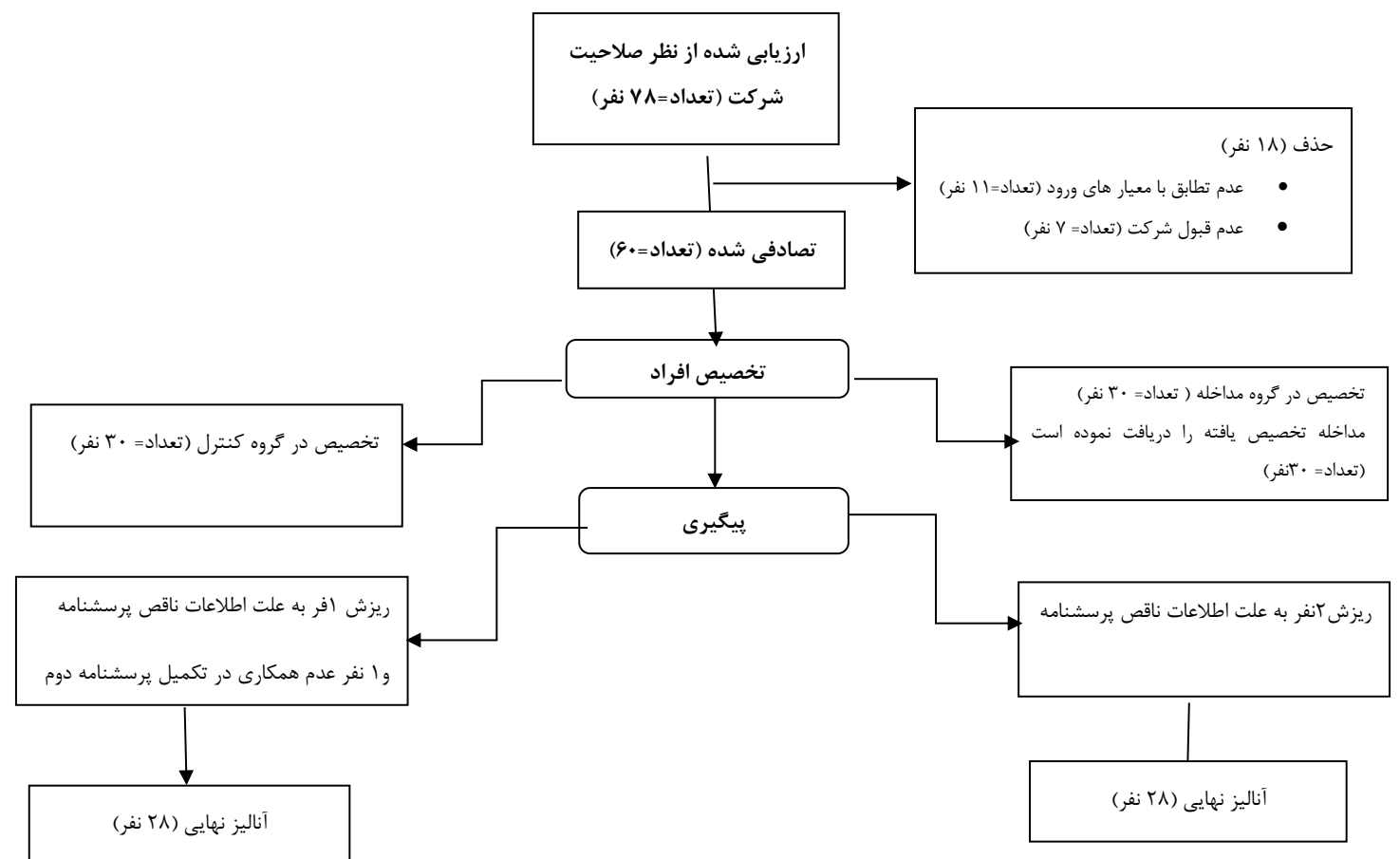


جدول ا. مقايسه اطلاعات فردى و بيمارى واحدهاى مورديثوهش در دو كروه تصويرسازى ذهنى و كنترل

\begin{tabular}{|c|c|c|c|c|}
\hline \multirow{3}{*}{$\begin{array}{c}P \\
\text { chi-Square } \\
.18 .\end{array}$} & \multicolumn{2}{|c|}{ ميانكين \انحراف معيار } & \multirow{2}{*}{\multicolumn{2}{|c|}{ متغير }} \\
\hline & كروه كثترل & كروه تصويرسازى ذهنى & & \\
\hline & $M / q \pm I F / M$ & $r \cdot / A \pm I V / r A$ & سال & سن \\
\hline \multirow{2}{*}{$\begin{array}{c}P \\
\text { chi-Square }\end{array}$} & \multicolumn{2}{|c|}{ درصد } & \multirow{2}{*}{\multicolumn{2}{|c|}{ متغير }} \\
\hline & كَووه كترل & كروه تصويرسازى ذهنى & & \\
\hline \multirow{2}{*}{.$/ V \&$} & $r \cdot(M / P)$ & $M I(V \Delta)$ & هرد & \multirow{2}{*}{ 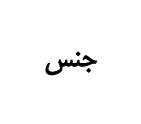 } \\
\hline & $A(Y N E)$ & $V(Y \Delta)$ & is & \\
\hline \multirow{2}{*}{$\cdot M$} & $r \cdot\left(M / I^{*}\right)$ & $19(9 V / 9)$ & متأهل & \multirow{2}{*}{ وضعيت تأهل } \\
\hline & $A(Y N E)$ & Q (IT/M) & مجرد & \\
\hline \multirow{2}{*}{.$/ 1 F$} & $M(V N E)$ & $\operatorname{IV}(\varepsilon \cdot M)$ & شهر & \multirow{2}{*}{ مجل سكونت } \\
\hline & $\varepsilon(r) / F)$ & $11(r q / \pi)$ & ل اوستا & \\
\hline \multirow{2}{*}{$\cdot M r$} & $9(\pi T / 1)$ & $\Lambda(Y N F)$ & دارد & \multirow{2}{*}{ سابقه بسترى } \\
\hline & $19(8 V / 9)$ & $r \cdot(V / / P)$ & ندارد & \\
\hline
\end{tabular}

(1)

كواريانس استفاده شد و اضطراب بعـد از مداخلـه بـا ثابت نكَه

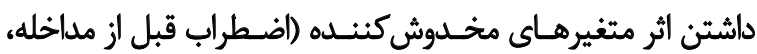

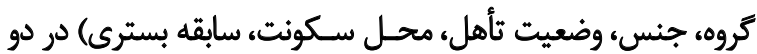
كروه بررسى شد.

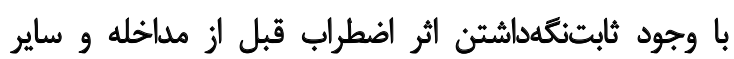

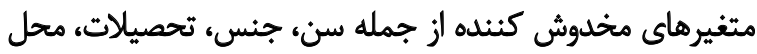

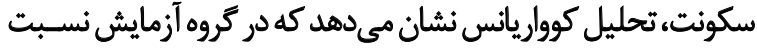

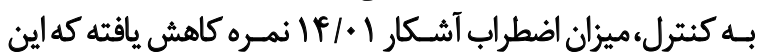

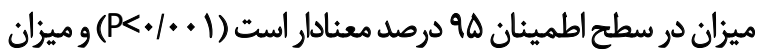

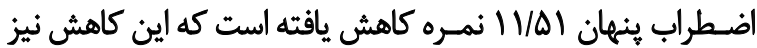

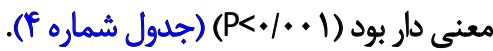

مطالعه به أبه

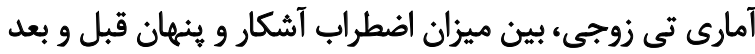

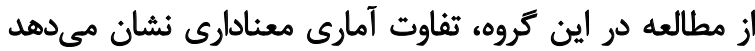

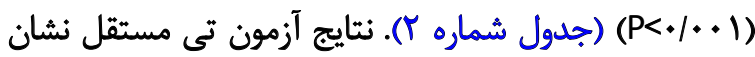

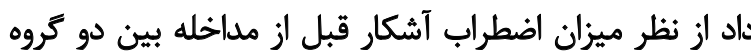

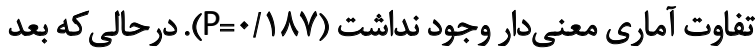

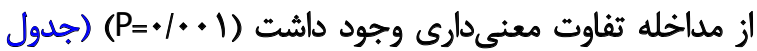

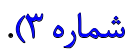

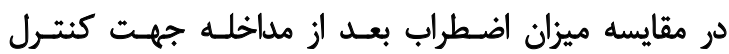

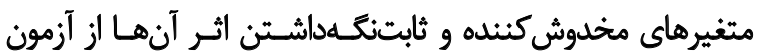

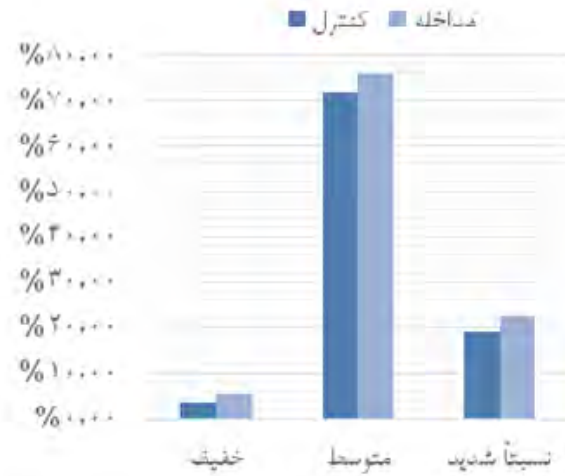


جدول r. مقايسه ميائكين نمره اضطراب واحدهاي يثروه در كروه تصوير سازى ذهنى و كنترل

\begin{tabular}{|c|c|c|c|c|}
\hline $\mathbf{P}$ & بعد از مداخله & قبل از مداخله & \multirow{2}{*}{ 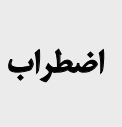 } & \multirow{2}{*}{ 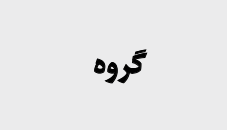 } \\
\hline تي زوجى & \multicolumn{2}{|c|}{ ميانغين土|نحراف معيار } & & \\
\hline $\mathrm{P}<\cdot 1+\cdot 1$ & $\pi / * V \pm r / \mu^{\prime}$ & PI/RTEE/VO & أشكار & \\
\hline$P<* / . .1$ & TNIFEV/NE & MTRTEQVAV & ت بنهان & كروه تصويرسازى ذهنى \\
\hline$P<\bullet / \bullet+1$ & $p 4 / 8 . \pm 8 / f 4$ & $r a n) \pm F / \Delta$ & آشكار & \\
\hline $\mathrm{P}<+/+* 1$ & TNFTEV/TI & rq/8N $\pm N F q$ & ينهان & \\
\hline
\end{tabular}

(3)

جدول r. ميانكين اضطراب آشكار قبل و بعد از مداخله در دو كروه تصويرسازى ذهنى و كنترل

\begin{tabular}{|c|c|c|c|}
\hline \multirow{2}{*}{ مستثل } & \multicolumn{2}{|c|}{ مياتكين \$|نحراف معيار } & \multirow{2}{*}{ اضشطراب أشيّكار } \\
\hline & كروه كثترل & كروه تصوير سازى ذهنى & \\
\hline$P=+/ M Y$ & $r q / V I \pm f / a 1$ & $P I / F Y \pm E / V A$ & قبل ازمداخله \\
\hline $\mathrm{P}<+/++1$ & $R e / g+ \pm g / p F$ & $M /+V \pm \psi / \mu 1$ & بعد از مداخله \\
\hline
\end{tabular}

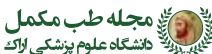

از عمل بر بيماران مؤثر است. نتايج مطالعه كونزالس و همكاران

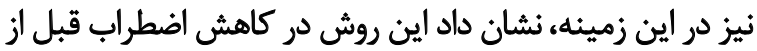

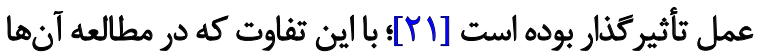

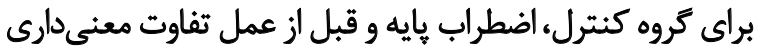

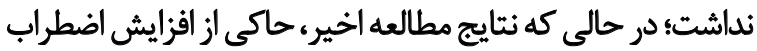

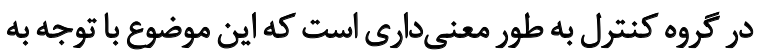

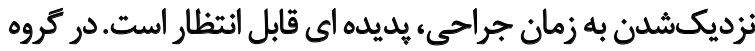

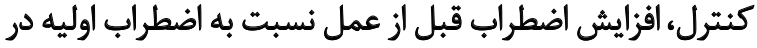

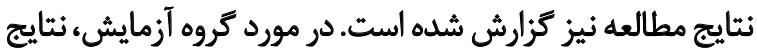

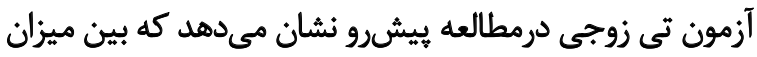

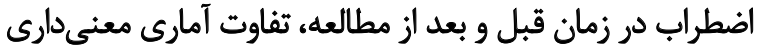

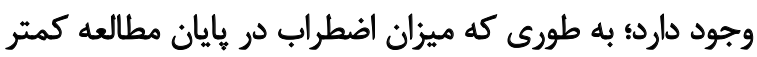

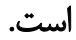

هديجى بلاسى و همكاران نيز در مطالعهاى مرورى،

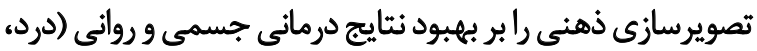

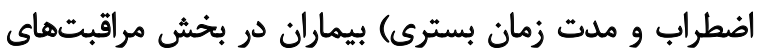

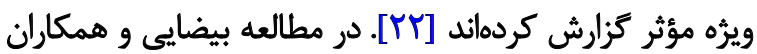

يافتههاى مطالعه حاضر نشان ميدهد انجام يك جلسه

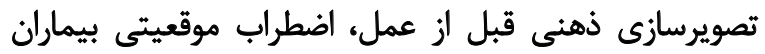

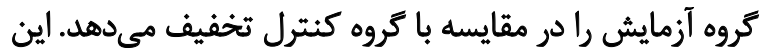

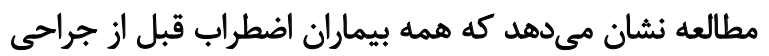

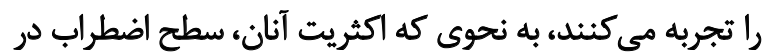

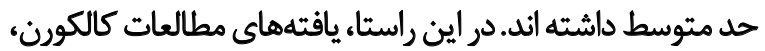

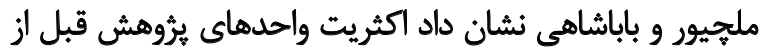

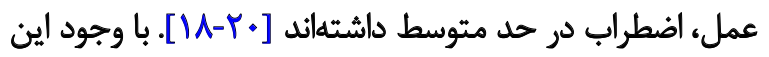

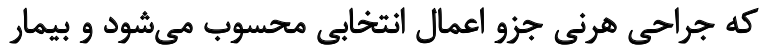

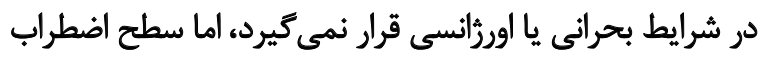

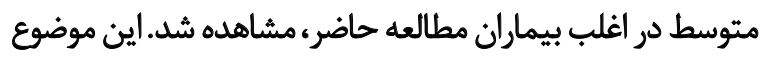

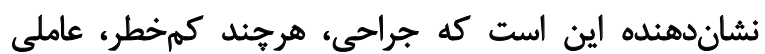
اضطرابآور و استرسزا محسوب ميى استود.

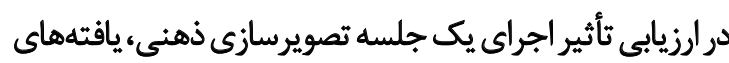

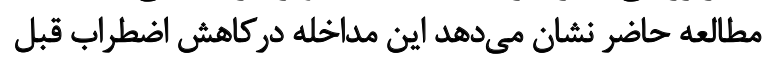

جدول F. متغيرهاي تأثير كذار در محاسبه اضطراب آشكار و ينهان بعد از مداخله بر اساس تحليل كوواريانس

\begin{tabular}{|c|c|c|c|}
\hline B & $\mathbf{t}$ & $\mathbf{P}$ & متغير \\
\hline$-1 F / .1$ & $-r \Delta / \Delta 1$ & $P<+1++1$ & اضطراب أشكار بعد مداخله \\
\hline$-|1 / \Delta|$ & $-2 / 49$ & $\mathrm{P}<+1 .+1$ & اضطراب بنهان بعد مداخله \\
\hline
\end{tabular}

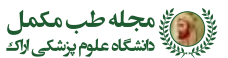




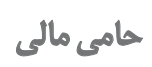

حامى مالى اين يُروهش دانشكاه علوميزشكى سبزوار است.

$$
\text { مشاركت نويسند مكان }
$$

در اين ثئوهش تنظيم و نمونه گيرى و تدوين مقاله بر عهله

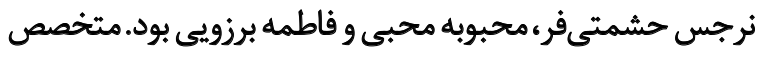

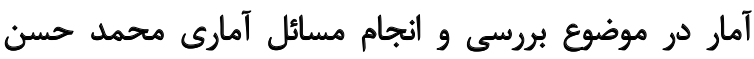

$$
\text { رخشانى بود. }
$$

$$
\text { تعارض مناقع }
$$

بنابر اظهار نويسندكان در اين مطالعه تضاد منافع وجود ندارد.

$$
\text { تشكر و قدردانى }
$$

مدينوسيله از معاونت يرؤششى دانشكاه علوميزشكى سبزوار،

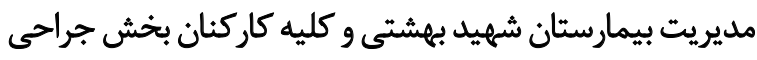

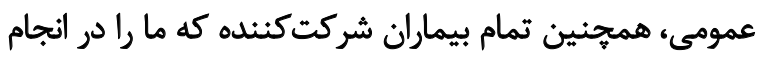
يثروهش يارى كردند، تشكر و قدردانى مى شئود
تصويرسازى ذهنى بر كاهش اضطراب، افسردگى و ثبات عالاتم

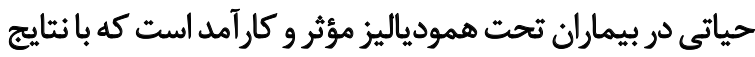

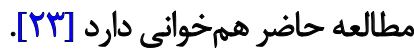

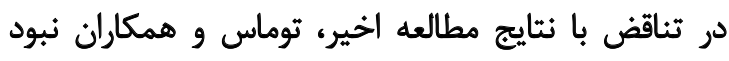

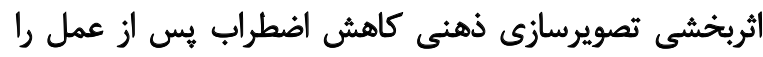

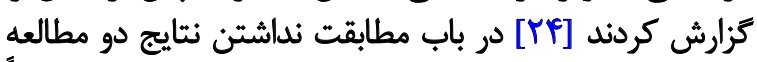

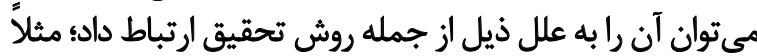

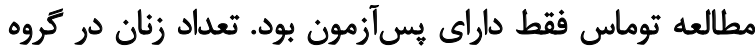

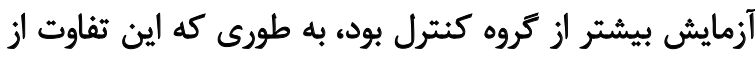

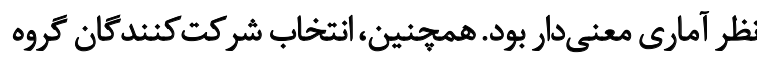

$$
\text { آزمايش غيرتصادفى بودي }
$$

نتايج مطالعه يورج و همكاران نيز نشاندهينده تفاوت نداشتين

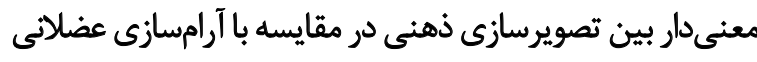

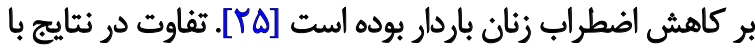

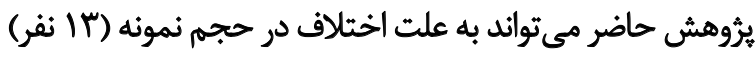

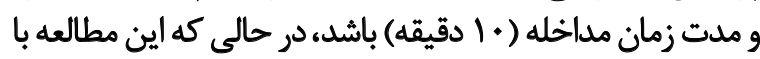

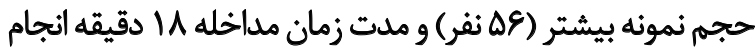

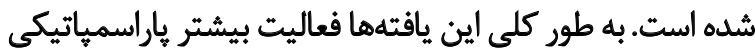

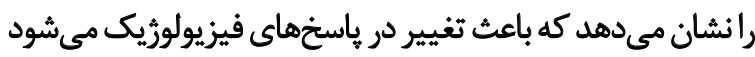

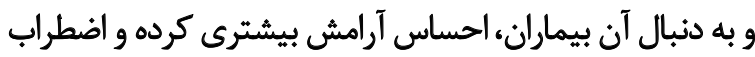

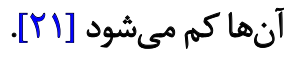

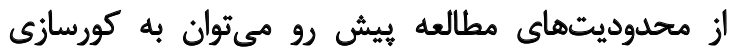

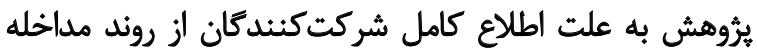

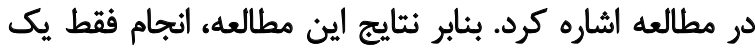

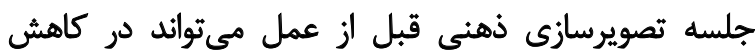

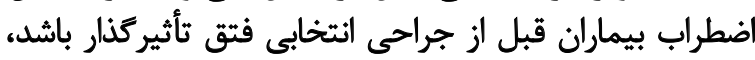

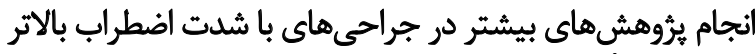

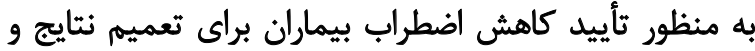
كاربردى بردن آن در بالين توصيه مي اضطي بيمارد.

$$
\text { تثيجلمَيرى }
$$

تصويرسازى ذهنى مي تواند به عنوان روشى آسان، ارزان و

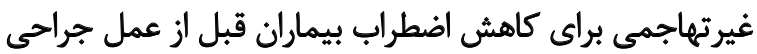
مورد استفاده قرار كيرد.

مالاحظات اخلاقي

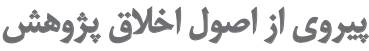

اين مقاله از طرح تحقيقاتى مصوب دانشكاه علوميزشكى

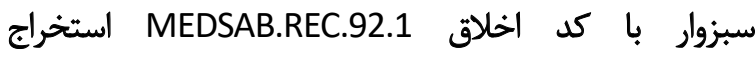
شد. همجنين در مركز ثبت كارآزمايى بالينى ايران با كد IRCT2013122315905N1 


\section{References}

[1] Sadati L, Golchini E, Nafar M, Khorasani B. [Digestive \& glandular surgical tecnology (Persian)]. 1st ed. Tehran: Jameenegar; 2011. http:// opac.nlai.ir/opac-prod/bibliographic/3627825

[2] Taylor C, Lillis C, LeMone P, Lynn P. Fundamentals of nursing: The art and science of nursing care. 6th ed. Philadelphia: Lippincott Williams \& Wilkins; 2001.

[3] Ramamohan D, Indira S, Sateesh S, Satish Kumar S, Bhandarkar P, Sahana Bhat NR, et al. Understanding preoperative anxiety in patients before elective surgical intervention. International Journal of Academic Medicine. 2018; 4(1):56-9. [DOI:10.4103/IJAM.IJAM_58_17]

[4] Britteon P, Cullum N, Sutton M. Association between psychological health and wound complications after surgery. British Journal of Surgery. 2017; 104(6):769-76. [DOI:10.1002/bjs.10474] [PMID]

[5] Akinsulore AD, Owojuyigbe AM, Faponle AF, Fatoye FO. Assessment of preoperative and postoperative anxiety among elective major surgery patients in a tertiary hospital in Nigeria. Middle East Journal of Anaesthesiology. 2015; 23(2):235-40. [PMID]

[6] World Health Organization. Patient safety: Making health care safer [Internet]. 2017 [Updated 2017 November]. Available from: https:// apps.who.int/iris/handle/10665/255507

[7] Kalogianni A, Almpani P, Vastardis L, Baltopoulos G, Charitos C, Brokalaki $\mathrm{H}$. Can nurse-led preoperative education reduce anxiety and postoperative complications of patients undergoing cardiac surgery? European Journal of Cardiovascular Nursing. 2016; 15(6):447-58. [DOI:10.1177/1474515115602678] [PMID]

[8] Binns-Turner PG, Wilson LL, Pryor ER, Boyd GL, Prickett CA. Perioperative music and its effects on anxiety, hemodynamics, and pain in women undergoing mastectomy. AANA Journal. 2011; 79(4):S21-S7. [PMID]

[9] Evans K, Jane Morrell C, Spiby H. Systematic review and meta-analysis of non-pharmacological interventions to reduce the symptoms of mild to moderate anxiety in pregnant women. Journal of Advanced Nursing. 2018; 74(2):289-309. [DOI:10.1111/jan.13456] [PMID]

[10] Schulz P, Hede V. Alternative and complementary approaches in psychiatry: Beliefs versus evidence. Dialogues in Clinical Neuroscience. 2018; 20(3):207-14. [PMID] [PMCID]

[11] Apóstolo JLA, Kolcaba K. The effects of guided imagery on comfort, depression, anxiety, and stress of psychiatric in patients with depressive disorders. Archives of Psychiatric Nursing. 2009; 23(6):403-11. [DOI:10.1016/j.apnu.2008.12.003] [PMID]

[12] Nasiri S, Akbari H, Tagharrobi L, Tabatabaee AS. The effect of progressive muscle relaxation and guided imagery on stress, anxiety, and depression of pregnant women referred to health centers. Journal of Education and Health Promotion. 2018; 7:41. [PMID] [PMCID]

[13] Stein TR, Olivo EL, Grand SH, Namerow PB, Costa J, Oz MC. A pilot study to assess the effects of aguided imagery audiotape interventionon psychological outcomes in patients undergoing coronary artery bypass graft surgery. Holistic Nursing Practice. 2010; 24(4):21322. [DOI:10.1097/HNP.0b013e3181e90303] [PMID]

[14] Jong M, Pijl A, de Gast H, Sjöling M. P02.128. The effects of guided imagery on preoperative anxiety and pain management in patients undergoing Laparoscopic Cholecystectomy in a multi-centre RCT study. BMC Complementary and Alternative Medicine. 2012; 12:P184. [DOI:10.1186/1472-6882-12-S1-P184] [PMCID]
[15] Wongkietkachorn A, Wongkietkachorn N, Rhunsiri P. Preoperative needs-based education to reduce anxiety, increase satisfaction, and decrease time spent in day surgery: A randomized controlled trial. World Journal of Surgery. 2018; 42(3):666-74. [DOI:10.1007/s00268017-4207-0] [PMID]

[16] Bagheri-Nesami M, Shorofi SA, Zargar N, Sohrabi M, Gholipour-Baradari A, Khalilian AR. The effects of foot reflexology massage on anxiety in patients following coronary artery bypass graft surgery: A randomized controlled trial. Complementary Therapies in Clinical Practice. 2014; 20(1):42-7. [DOI:10.1016/j.ctcp.2013.10.006] [PMID]

[17] Chamanzari H, Raffe S, Modarres Gheravi M, Ebrahimzade S. [Effect of guided imagery on orthopedic operations pain in traumatic patients (Persian)]. The Horizon of Medical Sciences. 2013; 19(2):117-22. http:// hms.gmu.ac.ir/article-1-1418-en.html

[18] Aghamohammadi Kalkhoran M, Karimollahi M. Religiousness and preoperative anxiety: A correlational study. Annals of General Psychiatry. 2007; 6:17. [DOI:10.1186/1744-859X-6-17] [PMID] [PMCID]

[19] Melchior LMR, dos Santos Soares Barreto RA, Prado MA, Caetano KAA, Bezerra ALQ, de Sousa TV. Predictors for moderate and serious preoperatory anxiety in hospitalized surgical patients. Enfermería Global. 2018; (52):86-96.

[20] Babashahi M, Kahangi LS, Babashahi F, Fayazi S. [Comparing the effect of massage aromatherapy and massage on anxiety level of the patients in the preoperative period: A clinical trial (Persian)]. Evidence Based Care. 2012; 2(2):19-28. http://ebcj.mums.ac.ir/article_395.html

[21] Gonzales EA, Ledesma RJA, McAllister DJ, Perry SM, Dyer CA, Maye JP. Effeets of guided imagery on postoperative outcomes in patients undergoing same-day surgical procedures: A randomized, single-blind study. AANA Journal. 2010; 78(3):181-8.

[22] Hadjibalassi M, Lambrinou E, Papastavrou E, Papathanassoglou E. The effect of guided imagery on physiological and psychological outcomes of adult ICU patients: A systematic literature review and methodological implications. Australian Critical Care. 2018; 31(2):73-86. [DOI:10.1016/j. aucc.2017.03.001] [PMID]

[23] Beizaee Y, Rejeh N, Heravi-Karimooi M, Tadrisi SD, Griffiths P, Vaismoradi $M$. The effect of guided imagery on anxiety, depression and vital signs in patients on hemodialysis. Complementary Therapies in Clinical Practice. 2018; 33:184-90. [DOI:10.1016/j.ctcp.2018.10.008] [PMID]

[24] Thomas KM, Sethares KA. Is guided imagery effective in reducing pain and anxiety in the postoperative total joint arthroplasty patient? Orthopaedic Nursing. 2010; 29(6):393-9. [DOI:10.1097/ NOR.0b013e3181f837f0] [PMID]

[25] Urech C, Fink N, Hoesli I, Wilhelm FH, Bitzer J, Alder J. Effects of relaxation on psychobiological wellbeing during pregnancy: A randomized controlled trial. Psychoneuroendocrinology. 2010; 35(9):1348-55. [DOI:10.1016/j.psyneuen.2010.03.008] [PMID] 